Article

\title{
Exploring the Excited-State Nonadiabatic Effects in the Semisaturated Planar Tetracoordinated Carbon Molecule $\mathrm{C}_{7} \mathrm{H}_{4}$
}

\author{
Chithra Mohan Jayakumari (D), Probal Nag, Sai Vamsi Krishna Isukapalli (D) and Sivaranjana Reddy Vennapusa * (D) \\ School of Chemistry, Indian Institute of Science Education and Research Thiruvananthapuram, \\ Maruthamala P.O., Vithura, Thiruvananthapuram 695551, India; chithramj17@iisertvm.ac.in (C.M.J.); \\ probalnag17@iisertvm.ac.in (P.N.); vamsi18@iisertvm.ac.in (S.V.K.I.) \\ * Correspondence: siva@iisertvm.ac.in
}

Citation: Jayakumari, C.M.; Nag, P.; Isukapalli, S.V.K.; Vennapusa, S.R

Exploring the Excited-State

Nonadiabatic Effects in the Semisaturated Planar

Tetracoordinated Carbon Molecule

$\mathrm{C}_{7} \mathrm{H}_{4}$. Atoms 2022, 10, 10.

https://doi.org/10.3390/

atoms 10010010

Academic Editors: Venkatesan S.

Thimmakondu and Krishnan

Thirumoorthy

Received: 22 December 2021

Accepted: 15 January 2022

Published: 19 January 2022

Publisher's Note: MDPI stays neutral with regard to jurisdictional claims in published maps and institutional affiliations.

Copyright: () 2022 by the authors Licensee MDPI, Basel, Switzerland. This article is an open access article distributed under the terms and conditions of the Creative Commons Attribution (CC BY) license (https:/ / creativecommons.org/licenses/by/ $4.0 /)$.

\begin{abstract}
We theoretically study the nonadiabatic relaxation dynamics of low-lying singlet excitedstates of semisaturated planar tetracoordinated carbon molecule, $\mathrm{C}_{7} \mathrm{H}_{4}$. This molecule possesses a stable $C_{2 v}$ ground-state equilibrium geometry. The three low-lying singlet states, $S_{1}, S_{2}$ and $S_{3}$, lie in the energy gap of about $1.2 \mathrm{eV}$. The potential energy surfaces constructed within the quadratic vibronic coupling formalism reveal multiple conical intersections in the Franck-Condon region. Upon photoexcitation to $S_{3}$, the wavepacket decays rapidly to lower states via these conical intersections. We also observe the wavepacket transfer to $\mathrm{S}_{3}$ during the initial wavepacket evolution on lower states, suggesting the nonadiabatic behavior of photoexcited planar $\mathrm{C}_{7} \mathrm{H}_{4}$.
\end{abstract}

Keywords: excited-states; nonadiabatic dynamics; relaxation pathways

\section{Introduction}

The ongoing research in planar tetracoordinated carbon $(\mathrm{ptC})$ for over five decades has opened a new era in the chemistry of carbon. Molecules containing ptC or even higher coordination have been successfully suggested and synthesized. The deviation from the long-established concept of tetrahedral tetracoordinate carbon by van't Hoff [1] and Le Bel [2] has raised the curiosity of researchers. Designing systems containing a planar tetracoordinated carbon center has remained a challenging task. The lone pair of electrons on the central ptC and the electron-deficient 3-centered 2-electron bonds make ptC unstable. The concept of ptC was first introduced by Monkhorst in 1968 [3]. Subsequently, using a planar methane model, Hoffmann and coworkers proposed strategies for stabilizing planar tetracoordinated carbon arrangements by electronic effects as well as steric effects $[4,5]$.

Using those strategies, a number of molecules were proposed theoretically [6-16], though only a few were identified in the laboratory [17-21]. Merino and coworkers have reported a series of semisaturated $\mathrm{ptC}$ candidates containing cyclic hydrocarbons, which were created by combining $\mathrm{C}_{5}^{2-}$ moiety with saturated hydrocarbon fragments [22]. These were the first semisaturated cycles, containing a ptC stabilized only by electronic factors. The authors analyzed various ground-state properties, and they inferred that the multicentric nature of the bonding within the $\mathrm{C}_{5}^{2-}$ skeleton and the resulting electron delocalization provides stability to these molecules.

Although several studies have focussed on the various ground-state properties of $\mathrm{ptCs}$, the excited-state properties of these molecules remain unexplored. In the present work, we focus on elucidating the excited-state dynamics of the semisaturated $\mathrm{ptC}, \mathrm{C}_{7} \mathrm{H}_{4}$ (tricyclo[4.1.0.0 $\left.{ }^{1,3}\right]$ hept-2,6-diene-2,7-diyl) (cf., Figure 1), using combined electronic structure computations and quantum dynamics simulations. We generate the potential energy surfaces (PESs) of low-lying singlet excited electronic states within the quadratic vibronic coupling (QVC) framework. Subsequently, we perform quantum-mechanical wavepacket simulations within the well-established multidimensional configurational time-dependent Hartree (MCTDH) method. Finally, we analyze the electronic populations and reduced 
nuclear densities and various stationary points of PESs to gain insights into the excited-state relaxation decay channels of the ptC molecule, $\mathrm{C}_{7} \mathrm{H}_{4}$.

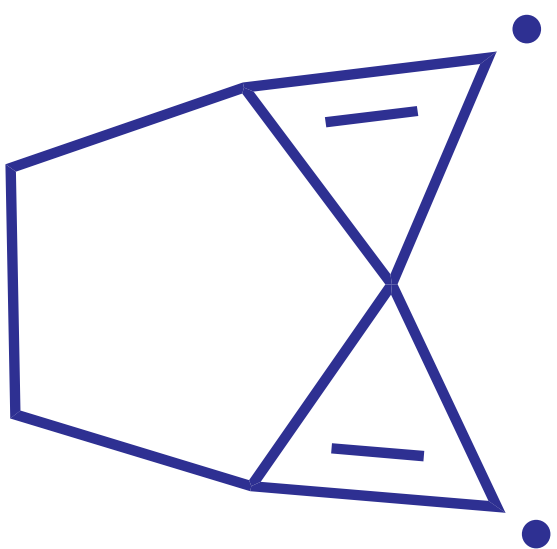

Figure 1. Structure of $\mathrm{C}_{7} \mathrm{H}_{4}$.

\section{Theoretical Framework}

\subsection{Electronic Structure Calculations}

We perform the optimization and frequency computations associated with the groundstate equilibrium geometry of $\mathrm{C}_{7} \mathrm{H}_{4}$ within the density functional theory (DFT) using gas-phase conditions. These computations use the B3LYP [23]/6-311++G(d,p) level of theory. For excited-state computations, we rely on the time-dependent variant of DFT (TDDFT). We obtain vertical excitation energies and corresponding oscillator strengths using the long-range corrected $\omega$ B97XD functional $(0.22 \mathrm{HF}$ at short-range and $1.00 \mathrm{HF}$ at long-range) [24] in combination with $6-311++\mathrm{G}(\mathrm{d}, \mathrm{p})$ basis set. All the calculations are done using Gaussian 16 program package [25].

To verify the suitability of the selected functional, we have done benchmarking calculations of excited-states with various quantum chemical methods. Accordingly, we employ time-dependent variants of B3LYP (containing 0.20 Hartree-Fock (HF) exchange), CAMB3LYP [26] (comprises of 0.19 HF exchange at short-range, and $0.65 \mathrm{HF}$ at long-range), LC- $\omega$ PBE [27] (no HF exchange at short-range and $1.00 \mathrm{HF}$ at long-range) and M06-2X (comprises of $0.54 \mathrm{HF}$ exchange) [28]. We use the $6-311++\mathrm{G}(\mathrm{d}, \mathrm{p})$ basis set for these computations. Further, we also evaluate excited-state energies using post-HF methods: equation of motion coupled cluster with single and double excitations (EOM-CCSD) method [29,30], resolution-of-the-identity second-order approximate coupled-cluster singles and doubles (RI-CC2) method [31], and algebraic diagrammatic construction method to second-order (ADC(2)) method [32]. These computations use the correlation consistent polarized valence double zeta (cc-pVDZ) basis set. The geometry optimized at the B3LYP/6-311++G(d,p) level of theory was used for all the above computations. We employ Gaussian 16 software package for TDDFT and EOM-CCSD computations and TURBOMOLE 7.4 software [33] for ADC(2) and RI-CC2 computations.

\subsection{Vibronic Hamiltonian}

Based on the computed vertical excitation energies, we are interested in studying the excited-state dynamics of the first three singlet excited-states of $\mathrm{C}_{7} \mathrm{H}_{4}$. For this, we construct a $3 \times 3$ vibronic Hamiltonian based on the well-established QVC approach [34]. The Hamiltonian can be expressed as: 


$$
\begin{gathered}
\mathcal{H}=\left(\mathrm{T}_{N}+\mathrm{V}_{0}\right) \mathbf{1}_{3}+ \\
\left(\begin{array}{ccc}
\mathrm{E}_{\mathrm{S}_{1}}^{0}+\sum_{i \in a_{1}} \kappa_{i}^{\left(\mathrm{S}_{1}\right)} \mathrm{Q}_{i}+\frac{1}{2} \sum_{i \in a_{1}} \gamma_{i}^{\left(\mathrm{S}_{1}\right)} \mathrm{Q}_{i}{ }^{2} & \sum_{i \in a_{1}} \lambda_{i}^{\left(\mathrm{S}_{1}-\mathrm{S}_{2}\right)} \mathrm{Q}_{i} & \sum_{j \in a_{2}} \lambda_{j}^{\left(\mathrm{S}_{1}-\mathrm{S}_{3}\right)} \mathrm{Q}_{j} \\
\text { h.c. } & \mathrm{E}_{\mathrm{S}_{2}}^{0}+\sum_{i \in a_{1}} \kappa_{i}^{\left.\mathrm{S}_{2}\right)} \mathrm{Q}_{i}+\frac{1}{2} \sum_{i \in a_{1}} \gamma_{i}^{\left(\mathrm{S}_{2}\right)} \mathrm{Q}_{i}{ }^{2} & \sum_{j \in a_{2}} \lambda_{j}^{\left(\mathrm{S}_{2}-\mathrm{S}_{3}\right)} \mathrm{Q}_{j} \\
\mathrm{E}_{\left.\mathrm{S}_{3}\right)}^{0}+\sum_{i \in a_{1}} \kappa_{i}^{\left(\mathrm{S}_{3}\right)} \mathrm{Q}_{i}+\frac{1}{2} \sum_{i \in a_{1}} \gamma_{i}^{\left(\mathrm{S}_{3}\right)} \mathrm{Q}_{i}{ }^{2}
\end{array}\right)
\end{gathered}
$$

Here, $\mathrm{T}_{N}$ and $\mathrm{V}_{0}$ are the ground-state kinetic energy and potential energy operators and are expressed in terms of the dimensionless normal coordinate (Q) within the harmonic approximation. $E_{\mathrm{m}}^{0}$ represents the vertical excitation energy of the excited-states (for $S_{\mathrm{m}}$, $\mathrm{m}=1,2$ and 3). $\kappa_{i}$ and $\gamma_{i}$ denotes the linear and quadratic intrastate coupling parameters, respectively, along the totally symmetric vibrational mode, $i . \lambda_{j}$ is the interstate coupling parameter along the non-totally symmetric vibrational mode, $j$. The interstate coupling constants between states of same symmetry $\left(S_{1}\right.$ and $\left.S_{2}\right)$ are computed along the totally symmetric vibrational mode, $a_{1}$, and is represented as $\lambda_{i}$. These coupling parameters are evaluated using the following expressions:

$$
\begin{gathered}
\kappa^{\left(\mathrm{S}_{\mathrm{m}}\right)}=\left.\frac{\partial V_{\mathrm{S}_{\mathrm{m}}}}{\partial \mathrm{Q}_{i}}\right|_{\mathrm{Q}_{0}, \quad i \in a_{1}} \\
\gamma^{\left(\mathrm{S}_{\mathrm{m}}\right)}=\left.\frac{\partial^{2} V_{\mathrm{S}_{\mathrm{m}}}}{\partial \mathrm{Q}_{i}{ }^{2}}\right|_{\mathrm{Q}_{0},} \quad i \in a_{1} \\
\lambda_{i / j}^{\left(\mathrm{S}_{\mathrm{m}}-\mathrm{S}_{\mathrm{n}}\right)}=\left[\frac{1}{8} \frac{\partial^{2}}{\partial \mathrm{Q}_{i / j}^{2}}\left|V_{\mathrm{S}_{\mathrm{m}}}(\mathrm{Q})-V_{\mathrm{S}_{\mathrm{n}}}(\mathrm{Q})\right|^{2}\right]_{\mathrm{Q}_{0}}^{1 / 2}, \quad i \in a_{1} ; j \in a_{2}
\end{gathered}
$$

where, $V_{\mathrm{S}_{\mathrm{m}}}$ and $V_{\mathrm{S}_{\mathrm{n}}}$ are the adiabatic potential energies of two different electronic states, $\mathrm{S}_{\mathrm{n}}$ and $S_{m}$, respectively; $Q_{0}$ represents the ground-state equilibrium geometry of the molecule.

\subsection{Dynamics Simulations}

We simulate the quantum nuclear dynamics of the singlet excited-states of $\mathrm{C}_{7} \mathrm{H}_{4}$ by using multiconfiguration time-dependent Hartree (MCTDH) approach [35-37] which is designed to solve the time-dependent Schrodinger equation for multidimensional dynamical systems. In this method, the nuclear wavefunction, $\Psi$, of a system with $f$ degrees of freedom $(\mathrm{DOF})$ is expressed as:

$$
\Psi\left(\mathrm{Q}_{1}, \ldots, \mathrm{Q}_{f}, t\right)=\sum_{i_{1}=1}^{n_{1}}, \ldots, \sum_{i_{f}=1}^{n_{f}} R_{i_{1}, \ldots, i_{f}}(t) \prod_{k=1}^{f} \phi_{i k}^{(k)}\left(\mathrm{Q}_{k}, t\right) .
$$

where $Q_{1}, \ldots, Q_{f}$ are the nuclear coordinates of vibrational modes. $R_{i_{1}, \ldots, i_{f}}$ and $\phi_{i k}^{(k)}$ denote the MCTDH expansion coefficients and single-particle functions (SPFs), respectively. $n_{k}$ represents the number of SPFs to describe the $k$-th DOF.

To reduce the memory requirement of the above treatment, we adopt a "mode combination" technique in which SPFs that can describe a set of DOF are used. The nuclear wavefunction can be then rewritten as a multi-configuration over $p$ generalized particles:

$$
\Psi\left(\mathrm{Q}_{1}^{\prime}, \mathrm{Q}_{2}^{\prime}, \ldots, \mathrm{Q}_{f}^{\prime}, t\right)=\sum_{j_{1}=1}^{n_{1}}, \ldots, \sum_{j_{p}=1}^{n_{p}} R_{j_{1}, \ldots, j_{p}}(t) \phi_{j_{1}}^{(1)}\left(\mathrm{Q}_{1}^{\prime}, t\right), \ldots, \phi_{j_{p}}^{(p)}\left(\mathrm{Q}_{p}^{\prime}, t\right)
$$

with

$$
\phi_{j}^{(k)}\left(\mathrm{Q}_{k}^{\prime}, t\right)=\phi_{j}^{(k)}\left(\mathrm{Q}_{1}, \mathrm{Q}_{2}, \ldots, \mathrm{Q}_{w}, t\right)
$$

where $\mathrm{Q}_{k}^{\prime}=\left(\mathrm{Q}_{1}, \mathrm{Q}_{2}, \ldots, \mathrm{Q}_{w}\right)$ represents the multidimensional coordinate for mode $k$. 
Out of the 28 degrees of freedom (27 vibrational modes and a set of electronic states) of $\mathrm{C}_{7} \mathrm{H}_{4}$, a total of $14\left(9 a_{1}\right.$ and $\left.5 a_{2}\right)$ modes were selected based on the excitation strength of the modes. It is to be noted that $S_{1}-S_{3}$ and $S_{2}-S_{3}$ couplings are along $a_{2}$ modes and coupling between $S_{1}$ and $S_{2}$, belonging to the same symmetry, are along $a_{1}$ modes. The nuclear wavepacket generated on $S_{3}$ PES has been propagated for $300 \mathrm{fs}$ with a time step of $1 \mathrm{fs}$. The diabatic electronic populations and nuclear densities are then extracted to study the internal conversion dynamics. The Heidelberg MCTDH code version 8.5 Revision 11 [38] is employed for these calculations. Details of MCTDH dynamics such as mode combination, primitive basis, and SPFs are given in Supplementary Materials.

\section{Results and Discussion}

\subsection{PESs and Conical Intersections}

We collect the vertical excitation energies, oscillator strengths and symmetries of lowlying singlet excited-states of $\mathrm{C}_{7} \mathrm{H}_{4}$ computed at different levels of theory in Table 1. All computational methods yield the same electronic symmetries for $S_{1}$ and $S_{2}$. We also note that all methods predict $B_{2}$ symmetry for $S_{3}$ except TD-B3LYP and TD-M06-2X. The latter two methods show $\mathrm{A}_{1}$ symmetry for this state. Concerning oscillator strengths, all methods predict a smaller value for $S_{1}$ than higher electronic states. Here, the important feature of Table 1 is the $S_{1}-S_{3}$ energy gap; all methods predict a gap of about $1.2 \mathrm{eV}$. As these states lie within this energy gap, one would expect the molecule to follow nonadiabatic behavior upon photoexcitation. As the vertical energies (as well as the oscillator strengths) of the excited-states computed at the $\omega$ B97XD/6-311++G(d,p) level of theory match well with the computationally expensive wavefunction methods, we perform all calculations using this method.

Table 1. Vertical excitation energies of $\mathrm{C}_{7} \mathrm{H}_{4}$ computed using different levels of theory. 6-311++G(d,p) is the basis set used for the TDDFT methods and cc-pVDZ is used for the wavefunction based methods.

\begin{tabular}{cccc}
\hline Methods & $\mathrm{S}_{\mathbf{1}}$ & $\mathrm{S}_{\mathbf{2}}$ & $\mathrm{S}_{\mathbf{3}}$ \\
\hline TD- $\omega$ B97XD & $4.3417\left(\mathrm{~B}_{1}, 0.001\right)$ & $4.8192\left(\mathrm{~B}_{1}, 0.043\right)$ & $5.5606\left(\mathrm{~B}_{2}, 0.049\right)$ \\
TD-B3LYP & $4.0221\left(\mathrm{~B}_{1}, 0.005\right)$ & $4.5325\left(\mathrm{~B}_{1}, 0.032\right)$ & $5.0136\left(\mathrm{~A}_{1}, 0.010\right)$ \\
TD-CAMB3LYP & $4.3389\left(\mathrm{~B}_{1}, 0.001\right)$ & $4.7960\left(\mathrm{~B}_{1}, 0.045\right)$ & $5.5310\left(\mathrm{~B}_{2}, 0.045\right)$ \\
TD-LC- $\omega$ PBE & $4.5102\left(\mathrm{~B}_{1}, 0.000\right)$ & $4.9985\left(\mathrm{~B}_{1}, 0.050\right)$ & $5.7237\left(\mathrm{~B}_{2}, 0.037\right)$ \\
TD-M06-2X & $4.2035\left(\mathrm{~B}_{1}, 0.000\right)$ & $4.5731\left(\mathrm{~B}_{1}, 0.039\right)$ & $5.4983\left(\mathrm{~A}_{1}, 0.004\right)$ \\
EOM-CCSD & $4.3121\left(\mathrm{~B}_{1}, 0.002\right)$ & $4.9658\left(\mathrm{~B}_{1}, 0.032\right)$ & $5.5197\left(\mathrm{~B}_{2}, 0.031\right)$ \\
ADC $(2)$ & $4.3239\left(\mathrm{~B}_{1}, 0.003\right)$ & $4.9202\left(\mathrm{~B}_{1}, 0.030\right)$ & $5.4091\left(\mathrm{~B}_{2}, 0.042\right)$ \\
RICC2 & $4.2634\left(\mathrm{~B}_{1}, 0.004\right)$ & $4.8697\left(\mathrm{~B}_{1}, 0.027\right)$ & $5.3844\left(\mathrm{~B}_{2}, 0.046\right)$ \\
\hline
\end{tabular}

To explore the nuclear dependence of these electronic states, we plot adiabatic potential energy profiles (solid lines) against in-plane ring deformation vibrational coordinates $\left(\mathrm{Q}_{15}\right)$ in Figure 2. The ab initio energies (plus harmonic potential) are shown as filled circles in this figure. We observe that the potential energy profiles generated within the QVC approach reproduce the $a b$ initio energies quite well, confirming the suitability of the latter approach to study the dynamics happening within the Franck-Condon (FC) region. We observe multiple curve crossings between the excited- states of interest. Such crossings also occur in the other vibrational coordinate space, however those data are not shown here for brevity. It should be mentioned that these crossings would form the seam of conical intersection in the multidimensional space. Figure 3 depicts the conical intersections associated with the adiabatic PESs of $S_{1}, S_{2}$, and $S_{3}$ states along the coordinates of ring deformation $\left(Q_{15}\right)$ and $\mathrm{C}=\mathrm{C}$ stretch $\left(\mathrm{Q}_{22}\right)$ vibrations. These vibrational modes are represented in Figure 4 . 


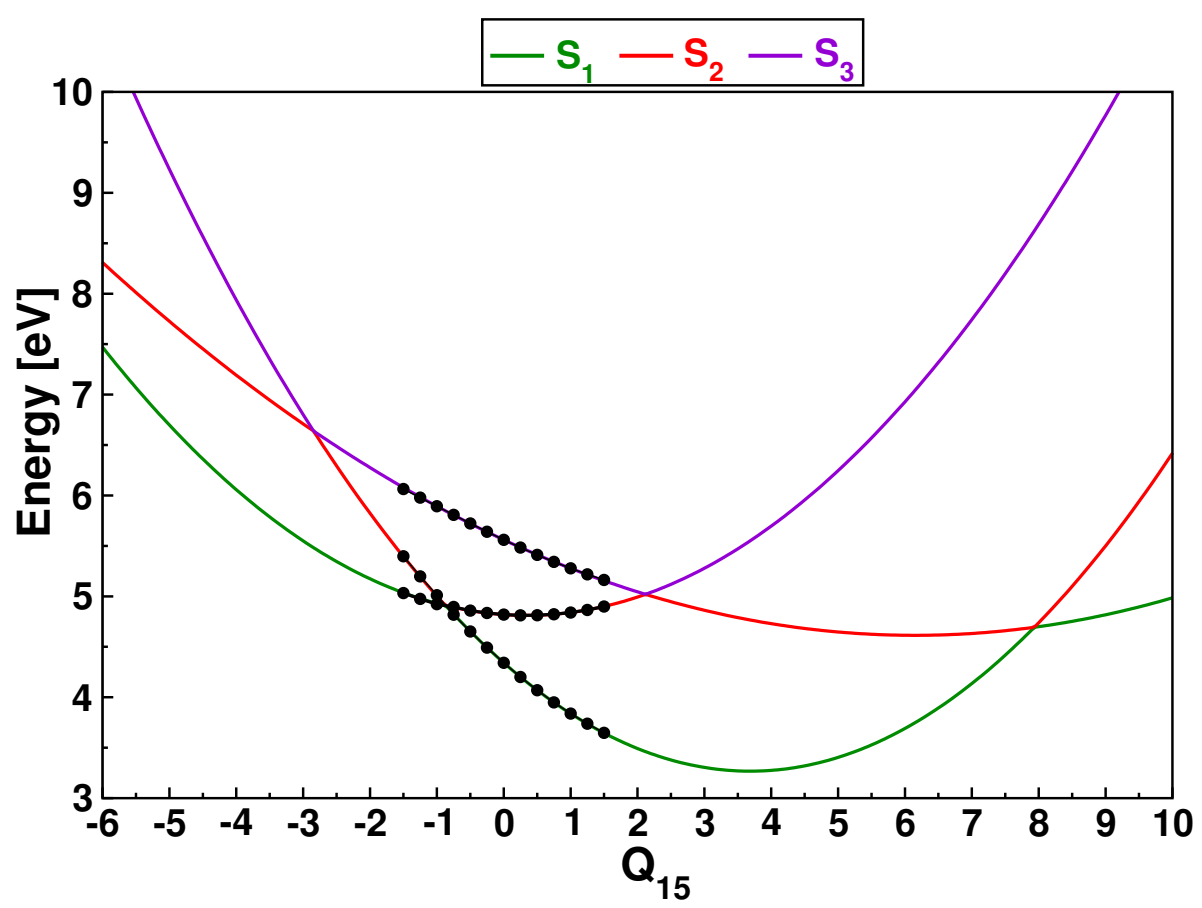

Figure 2. Computed adiabatic potential energy curves of along $\mathrm{Q}_{15}$ of $\mathrm{C}_{7} \mathrm{H}_{4}$ within the $\mathrm{QVC}$ approach. The solid circles represent the ab initio points plus ground-state harmonic potential energy.

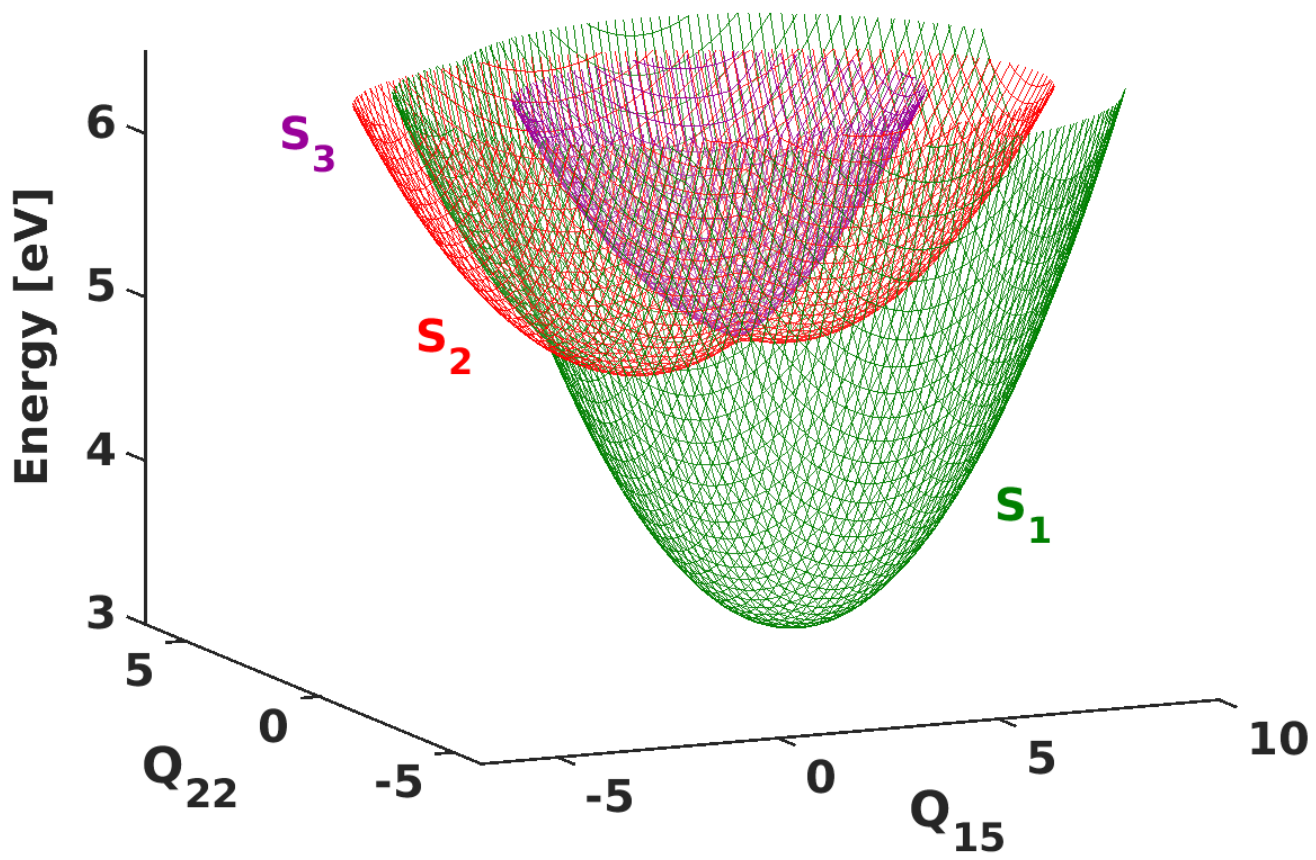

Figure 3. Adiabatic potential energy surfaces of $\mathrm{C}_{7} \mathrm{H}_{4}$ in the $\mathrm{Q}_{15}$ and $\mathrm{Q}_{22}$ vibrational space computed within the QVC approach. 


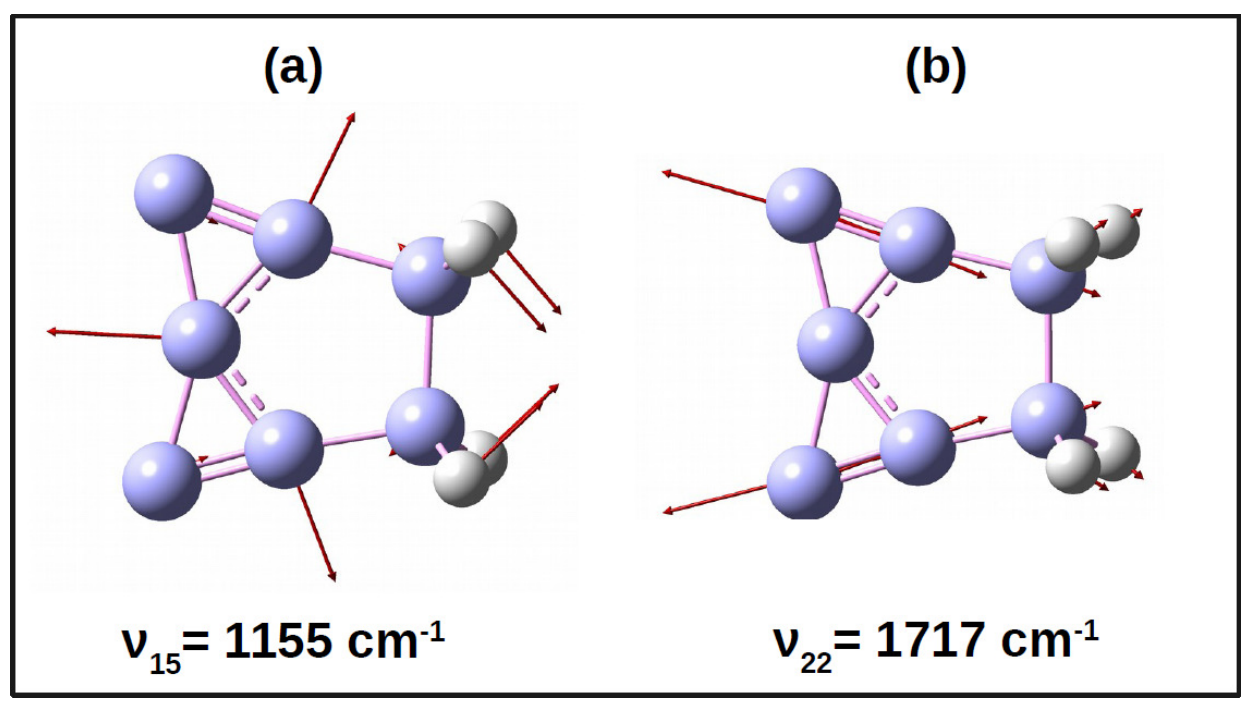

Figure 4. Schematic representation of the (a) in-plane ring deformation $\left(Q_{15}\right)$ and $(\mathbf{b}) C=C$ stretch $\left(\mathrm{Q}_{22}\right)$ vibrational modes of $\mathrm{C}_{7} \mathrm{H}_{4}$. Harmonic frequencies obtained using B3LYP/6-311++G(d,p) level of theory are also shown.

We also evaluate two other important quantities: minimum energy conical intersection and equilibrium minimum of excited-states. These quantities are crucial to explain the wavepacket dynamics happening on the coupled $S_{1}-S_{2}-S_{3}$ PESs. Following expressions are employed to evaluate those quantities: [39]

$$
\mathrm{MECI}=\mathrm{a}+\frac{(\mathrm{b}-\mathrm{c})^{2}}{2 \mathrm{~d}}-\frac{1}{2} \sum_{i=1}^{N} \frac{\sigma_{i}^{2}}{\omega_{i}}
$$

where,

$$
\begin{aligned}
\mathrm{a} & =\frac{\mathrm{E}_{1}^{0}+\mathrm{E}_{2}^{0}}{2} \\
\mathrm{c} & =\frac{\mathrm{E}_{2}^{0}-\mathrm{E}_{1}^{0}}{2} \\
\mathrm{~b} & =\sum_{i=1}^{N} \frac{\delta_{i} \sigma_{i}}{\omega_{i}} \\
\mathrm{~d} & =\sum_{i=1}^{N} \frac{\delta_{i}^{2}}{\omega_{i}} \\
\sigma_{i} & =\frac{\kappa_{i}^{(2)}+\kappa_{i}^{(1)}}{2} \\
\delta_{i} & =\frac{\kappa_{i}^{(2)}-\kappa_{i}^{(1)}}{2} \\
\mathrm{E}_{m}^{\min } & =\mathrm{E}_{m}^{0}-\frac{1}{2} \sum_{i=1}^{N} \frac{\kappa_{i}^{2}}{\omega_{i}}
\end{aligned}
$$

where $\omega_{i}$ is the harmonic frequency of the $i$ th totally symmetric vibrational mode and $N$ is the total number of such modes.

The molecule might display unexpected nonradiative decay dynamics due to the presence of accessible conical intersections within the FC region (cf., Table 2). For instance, the $S_{2}-S_{3}$ conical intersection lies $\sim 0.5 \mathrm{eV}$ below the FC point of $S_{3}$ (cf., Tables 1 and 2). Hence, upon excitation to $S_{3}$, the molecule can, in principle, decay rapidly via this conical intersection. We expect this intersection point and the $S_{1}-S_{3}$ conical intersection, 
that lies slightly above $(\sim 0.1 \mathrm{eV})$ the $\mathrm{FC}$ point of $S_{3}$, might play an important role in the decay dynamics.

Table 2. Relevant stationary points (state minima and MECI) on the coupled $\mathrm{S}_{1}-\mathrm{S}_{2}-\mathrm{S}_{3}$ PESs of $\mathrm{C}_{7} \mathrm{H}_{4}$.

\begin{tabular}{cc}
\hline Stationary Point & Energy (eV) \\
\hline$S_{1}^{\min }$ & 3.6039 \\
$S_{2}^{\min }$ & 4.7940 \\
$S_{3}^{\min }$ & 4.3006 \\
$\mathrm{~S}_{1} / S_{2}$ & 4.7970 \\
$S_{1} / S_{3}$ & 5.6376 \\
$S_{2} / S_{3}$ & 5.0552 \\
\hline
\end{tabular}

\subsection{Singlet Dynamics}

To investigate the fate of $\mathrm{C}_{7} \mathrm{H}_{4}$ in the higher excited singlet states, we perform dynamics simulations by launching the initial wavepacket at the FC point of the "bright" $\mathrm{S}_{3}$. Figure 5a collects the time-dependent electronic population profiles obtained from this wavepacket propagation calculation. We observe a rapid population transfer from $\mathrm{S}_{3}$ to $\mathrm{S}_{2}$. The wavepacket evolving on $S_{3}$ could easily access the $S_{2} / S_{3} \mathrm{MECI}(\sim 5.06 \mathrm{eV}$, cf., Table 2$)$ and thus promote rapid nonadiabatic population transfer.

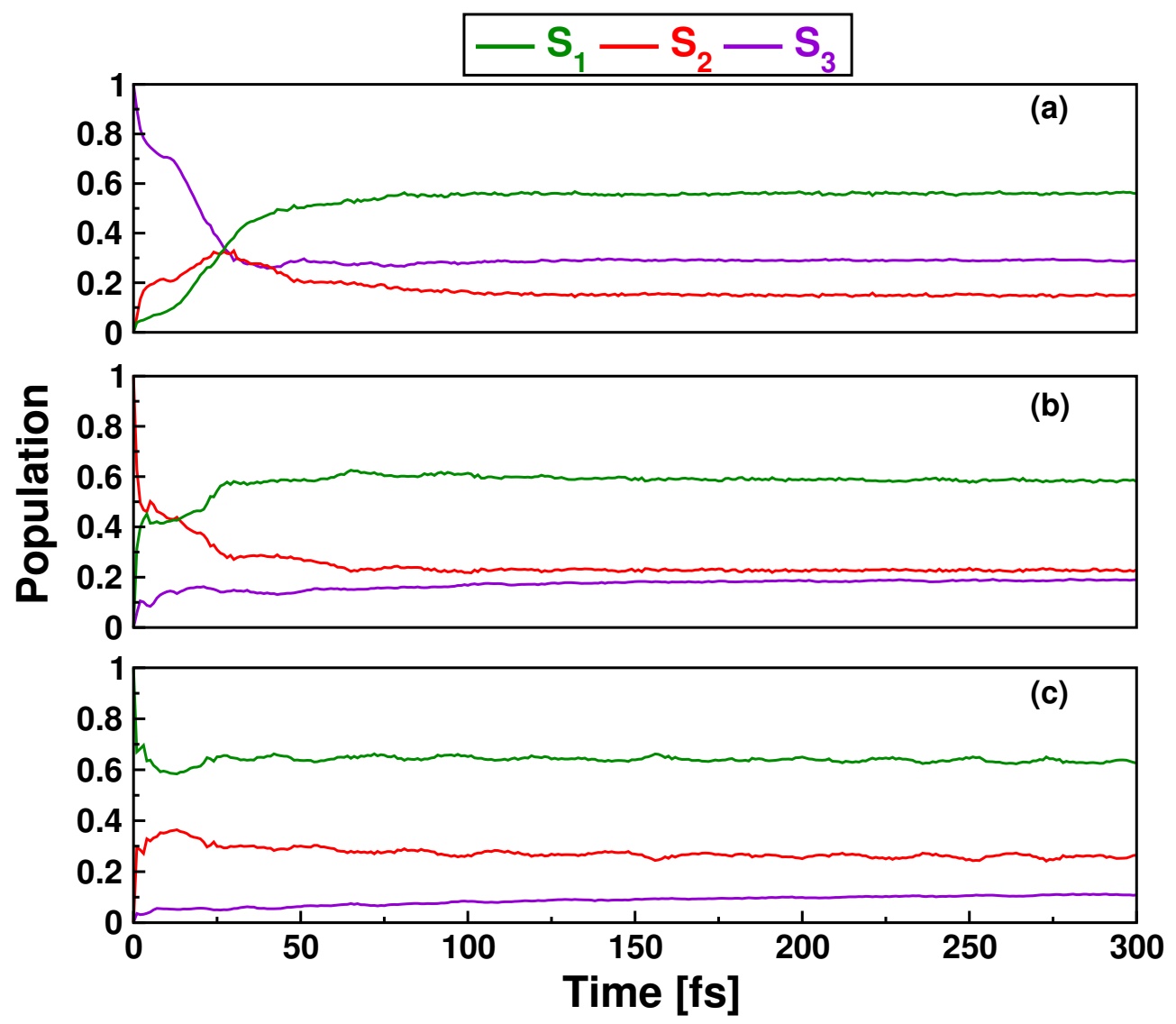

Figure 5. Diabatic electronic population profiles of singlet excited-states generated by propagation of initial wavepacket on (a) $\mathrm{S}_{3}$, (b) $\mathrm{S}_{2}$, and (c) $\mathrm{S}_{1}$ of $\mathrm{C}_{7} \mathrm{H}_{4}$.

We note that $\sim 70 \%$ depopulation of $S_{3}$ happens within the first 40 fs of propagation time. We observe a sharp rise in the $S_{1}$ population during this period. This sharp rise would emerge from the wavepacket decay via $S_{1}-S_{3}$ and $S_{1}-S_{2}$ conical intersections. Interestingly, the populations of the involved states remain almost unchanged after $50 \mathrm{fs}$. These 
observations suggest the remnant wavepacket localization at the respective equilibrium minimum of involved electronic states after the early nonadiabatic wavepacket transfer.

To further validate the nonadiabatic population transfer, we plot the reduced nuclear densities associated with the ring deformation mode, i.e., $\mathrm{Q}_{15}$, for three electronic states in Figure 6 . The density would be maximum on $S_{3}$ at $t=0$ fs as the wavepacket propagation starts on this state. We observe the rapid density reduction within $20 \mathrm{fs}$, and after that, the density remains unchanged until the end of propagation time. For $S_{2}$, the density accumulates rapidly after a few femtoseconds and reaches a maximum at about $20 \mathrm{fs}$. After that, the density reduces up to $50 \mathrm{fs}$ and remains unchanged till $300 \mathrm{fs}$. For $\mathrm{S}_{1}$, we observe a gradual increase of nuclear density, reaching a maximum value at about $20 \mathrm{fs}$. After that, the nuclear density on $S_{1}$ would remain unchanged up to $300 \mathrm{fs}$. From these observations, we infer that the wavepacket decay to $S_{1}$ would occur within a few tens of femtoseconds after photoexcitation to $S_{3}$.
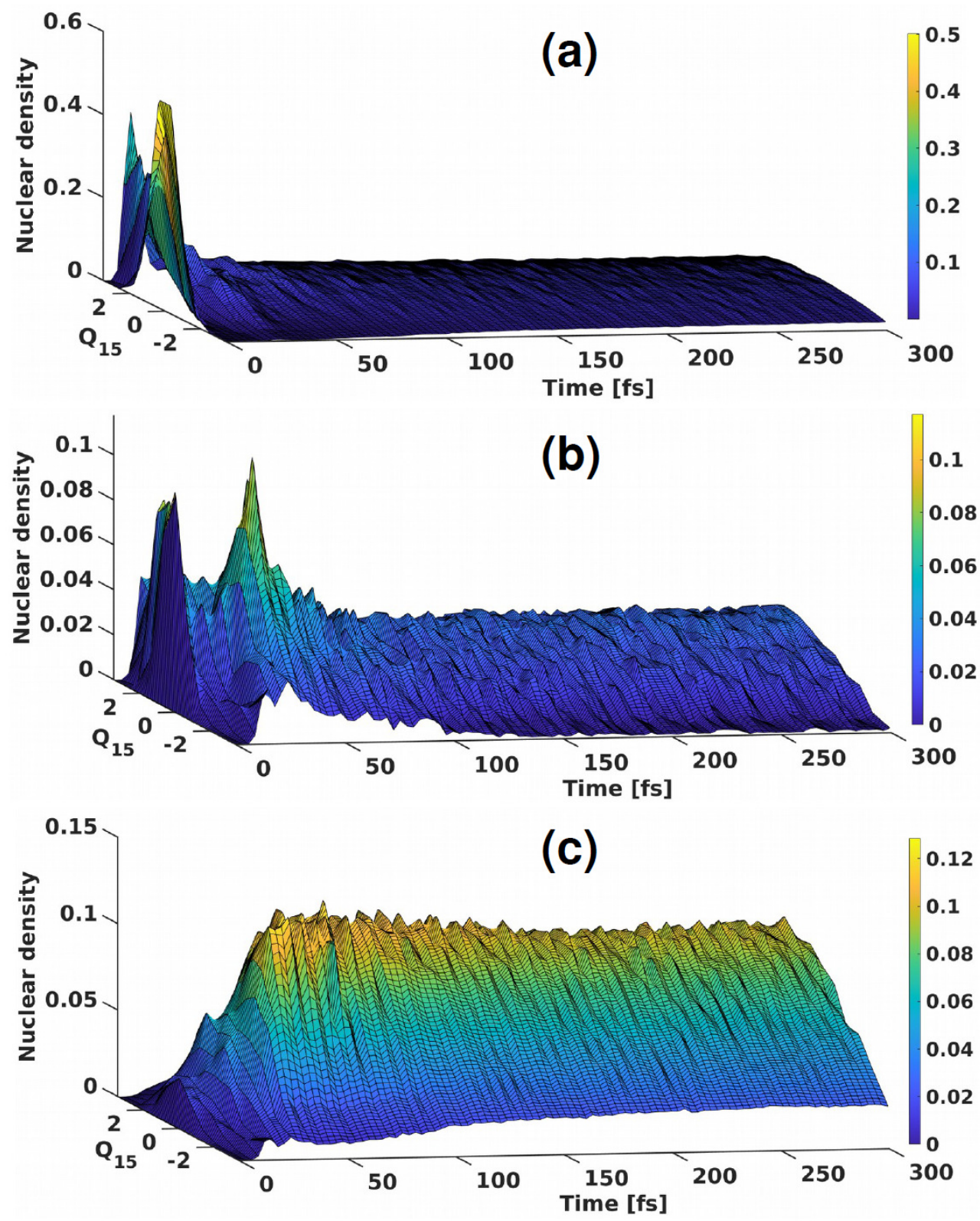

Figure 6. Nuclear densities variation of (a) $S_{3}$, (b) $S_{2}$, and (c) $S_{1}$ along $Q_{15}$ of $C_{7} H_{4}$, obtained by propagating initial wavepacket on the "bright" $S_{3}$ state. 
Next, we launch the initial wavepacket at the FC point of $S_{2}$ and $S_{1}$ to explore the decay dynamics of individual electronic states. The accessible $S_{1} / S_{2}$ MECI $(\sim 4.80 \mathrm{eV}$, cf., Table 2) combined with the high interstate coupling via $a_{1}$ modes results in an extremely rapid population transfer from $S_{2}$ to $S_{1}$ (cf., Figure $5 b$ ). The $S_{1}$ population reaches an asymptotic value of $\sim 0.6$ at the end of $300 \mathrm{fs}$. A minor population transfer to $S_{3}$ can also be seen, attributed to the strong nonadiabatic coupling between $S_{2}$ and $S_{3}$ states.

The wavepacket propagated on the $S_{1}$ PES shows an appreciable decay $(\sim 40 \%)$ to $S_{2}$ till the initial $20 \mathrm{fs}$ and further, remains constant (cf., Figure $5 \mathrm{c}$ ). Such population profile suggests that the molecule might be trapped at the equilibrium minimum of $S_{1}$, which is $\sim 0.7 \mathrm{eV}$ lower in energy from the $\mathrm{S}_{1} \mathrm{FC}$ point. The molecule could no longer access any conical intersections, resulting in no noticeable population transfer. Similar profile is also observed in the variation of nuclear densities (along $Q_{15}$ vibrational mode) across the excited-states with the wavepacket evolving on $\mathrm{S}_{1}$ (cf., Figure $\mathrm{S} 1$ in Supplementary Materials). It is noted that no state shows $100 \%$ decay within 300 fs. So dynamics simulations need to be conducted for a longer duration for the complete decay of the excited-states.

We note that the QVC model employed in this study would be helpful to gain insights into the FC dynamics. However, concerning the fluxional behavior of molecules containing $\mathrm{ptC}$, it is necessary to adopt a theoretical model that involves possible excited-state dissociation pathways. Identifying such dissociative pathways through experimental investigations can be complicated due to nonadiabatic events occurring in the FC region. However, one could rely on the high-level on-the-fly dynamics simulations to study the photoproducts of ptC molecules.

\section{Conclusions}

In the present work, we provide vital insights on the nonadiabatic relaxation dynamics of the semisaturated ptC molecule, $\mathrm{C}_{7} \mathrm{H}_{4}$. PESs constructed by employing QVC formalism revealed multiple conical intersections among the three singlet excited-states, $S_{1}, S_{2}$, and $S_{3}$. The molecule excited to the optically "bright" $S_{3}$ returns to $S_{1}$ on an ultrafast timescale via these conical intersections. Similarly, these crossing points also promote the wavepacket transfer to higher states $\left(S_{2}\right.$ and $\left.S_{3}\right)$ while the wavepacket is initially on $S_{1}$, demonstrating the nonadiabatic behavior of $\mathrm{C}_{7} \mathrm{H}_{4}$ irrespective of excitation energy. Although the present study has provided essential features of excited-state decay dynamics, a detailed quantum dynamics study using the multi-reference configuration interaction (MRCI) or multiconfigurational quasidegenerate perturbation theory (MCQDPT) PESs is planned to provide an accurate picture of the relaxation pathways of $\mathrm{C}_{7} \mathrm{H}_{4}$.

Supplementary Materials: The following supporting information can be downloaded at: https: / /www.mdpi.com/article/10.3390/atoms10010010/s1. Figure S1: Nuclear densities variation of the singlet excited states of $\mathrm{C}_{7} \mathrm{H}_{4}$ with the wavepacket evolving on $\mathrm{S} 1$ state, Table S1: Harmonic vibrational frequencies of $\mathrm{C}_{7} \mathrm{H}_{4}$ calculated at B3LYP/6-311++G(d,p) level of theory, Table S2: Groundstate (S0) equilibrium geometry of $\mathrm{C}_{7} \mathrm{H}_{4}$ optimized at B3LYP/6-311++G(d,p) level of theory, Table S3: Linear intrastate coupling parameters $(\kappa)$ for the singlet electronic states of $\mathrm{C}_{7} \mathrm{H}_{4}$ calculated at (TD) $\omega B$ B7XD/6-311++G(d,p) level of theory, Table S4: Quadratic intrastate coupling parameters $(\gamma)$ for the singlet electronic states of $\mathrm{C}_{7} \mathrm{H}_{4}$ calculated at (TD) $\omega \mathrm{B} 97 \mathrm{XD} / 6-311++\mathrm{G}(\mathrm{d}, \mathrm{p})$ level of theory, Table S5: Linear interstate coupling parameters $(\gamma)$ computed along a2 modes for the singlet electronic states of $\mathrm{C}_{7} \mathrm{H}_{4}$ calculated at (TD) $\omega$ B97XD/6-311++G(d,p) level of theory, Table S6: Linear interstate coupling parameters $(\gamma)$ computed along a1 modes for the singlet electronic states of $\mathrm{C}_{7} \mathrm{H}_{4}$ calculated at (TD) $\omega$ B97XD/6-311++G(d,p) level of theory, Table S7: MCTDH details of S1-S2-S3 vibronic dynamics of $\mathrm{C}_{7} \mathrm{H}_{4}$.

Author Contributions: Conceptualization, S.R.V.; methodology, S.R.V.; validation, S.R.V.; formal analysis, C.M.J., P.N. and S.V.K.I.; investigation, C.M.J., P.N. and S.V.K.I.; data curation, C.M.J., P.N. and S.V.K.I.; writing—original draft preparation, C.M.J.; writing—review and editing, S.R.V.; supervision, S.R.V. All authors have read and agreed to the published version of the manuscript.

Funding: This research received no external funding. 
Data Availability Statement: Data is contained within the article or supplementary material.

Acknowledgments: C.M.J. acknowledges Council of Scientific and Industrial Research (CSIR), Government of India, for doctoral fellowship. P.N. thanks Ministry of Higher Education, Government of India, for the fellowship under the Prime Minister's Research Fellows (PMRF) scheme. S.V.K.I. (DST/INSPIRE/IF170980) thanks Department of Science and Technology, New Delhi, for DSTINSPIRE fellowship. All authors gratefully acknowledge IISER TVM for computational resources.

Conflicts of Interest: The authors declare no conflict of interest.

\begin{tabular}{|c|c|}
\hline \\
\hline \multicolumn{2}{|c|}{$\begin{array}{l}\text { Abbreviations } \\
\text { The following abbreviations are used in this manuscript: }\end{array}$} \\
\hline ptC & Planar tetracoordinated carbon \\
\hline PES & Potential energy surface \\
\hline QVC & Quadratic vibronic coupling \\
\hline MCTDH & Multiconfiguration time-dependent hartree \\
\hline TDDFT & Time-dependent density functional theory \\
\hline EOM-CCSD & Equation of motion coupled cluster with single and double excitations \\
\hline RI-CC2 & $\begin{array}{l}\text { Resolution-of-the-identity second-order approximate coupled-cluster } \\
\text { singles and doubles }\end{array}$ \\
\hline $\operatorname{ADC}(2)$ & Algebraic diagrammatic construction method to second-order \\
\hline FC & Franck-Condon \\
\hline MECI & Minimum energy conical intersection \\
\hline
\end{tabular}

\section{References}

1. van't Hoff, J.H. A suggestion looking to the extension into space of the structural formulas at present used in chemistry, and a note upon the relation between the optical activity and the chemical constitution of organic compounds. Arch. Neerl. Sci. Exact Nat. 1874, 9, 445-454.

2. Le Bel, J. On the relations which exist between the atomic formulas of organic compounds and the rotatory power of their solutions. Bull. Soc. Chim. 1874, 22, 337-347.

3. Monkhorst, H.J. Activation energy for interconversion of enantiomers containing an asymmetric carbon atom without breaking bonds. Chem. Commun. 1968, 11, 1111-1112. [CrossRef]

4. Hoffmann, R.; Alder, R.W.; Wilcox, C.F. Planar tetracoordinate carbon. J. Am. Chem. Soc. 1970, 92, 4992-4993. [CrossRef]

5. Hoffmann, R. The theoretical design of novel stabilized systems. Pure Appl. Chem. 1971, 28, 181-194. [CrossRef]

6. McGrath, M.P.; Radom, L. Alkaplanes: a class of neutral hydrocarbons containing a potentially planar tetracoordinate carbon. J. Am. Chem. Soc. 1993, 115, 3320-3321. [CrossRef]

7. Lyons, J.E.; Rasmussen, D.R.; McGrath, M.P.; Nobes, R.H.; Radom, L. Octaplane: A saturated hydrocarbon with a remarkably low ionization energy leading to a cation with a planar tetracoordinate carbon atom. Angew. Chem. Int. Ed. Engl. 1994, 33, 1667-1668. [CrossRef]

8. Sorger, K.; Schleyer, P.v.R. Planar and inherently non-tetrahedral tetracoordinate carbon: A status report. J. Mol. Struct. Theochem 1995, 338, 317-346. [CrossRef]

9. Röttger, D.; Erker, G. Compounds Containing Planar-Tetracoordinate Carbon. Angew. Chem. Int. Ed. Engl. 1997, 36, 812-827. [CrossRef]

10. Radom, L.; Rasmussen, D.R. The planar carbon story. Pure Appl. Chem. 1998, 70, 1977-1984. [CrossRef]

11. Siebert, W.; Gunale, A. Compounds containing a planar-tetracoordinate carbon atom as analogues of planar methane. Chem. Soc. Rev. 1999, 28, 367-371. [CrossRef]

12. Rasmussen, D.R.; Radom, L. Planar-Tetracoordinate Carbon in a Neutral Saturated Hydrocarbon: Theoretical Design and Characterization. Angew. Chem. Int. Ed. 1999, 38, 2875-2878. [CrossRef]

13. Rasmussen, D.R.; Radom, L. Hemispiroalkaplanes: Hydrocarbon Cage Systems with a Pyramidal-Tetracoordinate Carbon Atom and Remarkable Basicity. Chem. A Eur. J. 2000, 6, 2470-2483. [CrossRef]

14. Priyakumar, U.D.; Sastry, G.N. A system with three contiguous planar tetracoordinate carbons is viable: a computational study on a C6H62 = isomer. Tetrahedron Lett. 2004, 45, 1515-1517. [CrossRef]

15. Perez, N.; Heine, T.; Barthel, R.; Seifert, G.; Vela, A.; Mendez-Rojas, M.A.; Merino, G. Planar tetracoordinate carbons in cyclic hydrocarbons. Org. Lett. 2005, 7, 1509-1512. [CrossRef]

16. Merino, G.; Méndez-Rojas, M.A.; Vela, A.; Heine, T. Recent advances in planar tetracoordinate carbon chemistry. J. Comput. Chem. 2007, 28, 362-372. [CrossRef]

17. Li, X.; Wang, L.S.; Boldyrev, A.I.; Simons, J. Tetracoordinated planar carbon in the Al4C-anion. A combined photoelectron spectroscopy and ab initio study. J. Am. Chem. Soc. 1999, 121, 6033-6038. [CrossRef] 
18. Li, X.; Zhang, H.; Wang, L.; Geske, G.; Boldyrev, A. ZUSCHRIFTEN-Pentaatomic Tetracoordinate Planar Carbon,(CAl4) 2-: A New Structural Unit and Its Salt Complexes Stichworter: Ab-initio-Rechnungen. Aluminium. Charge-Transfer Koordinationschemie. Angew.-Chem.-Ger. Ed. 2000, 112, 3776-3778. [CrossRef]

19. Wang, L.S.; Boldyrev, A.I.; Li, X.; Simons, J. Experimental observation of pentaatomic tetracoordinate planar carbon-containing molecules. J. Am. Chem. Soc. 2000, 122, 7681-7687. [CrossRef]

20. Li, X.; Zhai, H.J.; Wang, L.S. Photoelectron spectroscopy of pentaatomic tetracoordinate planar carbon molecules: CAl3Si- and CAl3Ge-. Chem. Phys. Lett. 2002, 357, 415-419. [CrossRef]

21. Xu, J.; Zhang, X.; Yu, S.; Ding, Y.h.; Bowen, K.H. Identifying the Hydrogenated Planar Tetracoordinate Carbon: A Combined Experimental and Theoretical Study of CAl4H and CAl4H-. J. Phys. Chem. Lett. 2017, 8, 2263-2267. [CrossRef] [PubMed]

22. Perez-Peralta, N.; Sanchez, M.; Martin-Polo, J.; Islas, R.; Vela, A.; Merino, G. Planar Tetracoordinate Carbons in Cyclic Semisaturated Hydrocarbons. J. Org. Chem. 2008, 73, 7037-7044. [CrossRef] [PubMed]

23. Becke, A.D. Density-functional thermochemistry. III. The role of exact exchange. J. Chem. Phys. 1993, 98, 5648-5652. [CrossRef]

24. Chai, J.D.; Head-Gordon, M. Long-range corrected hybrid density functionals with damped atom-atom dispersion corrections. Phys. Chem. Chem. Phys. 2008, 10, 6615-6620. [CrossRef]

25. Frisch, M.J.; Trucks, G.W.; Schlegel, H.B.; Scuseria, G.E.; Robb, M.A.; Cheeseman, J.R.; Scalmani, G.; Barone, V.; Petersson, G.A.; Nakatsuji, H.; et al. Gaussian 16 Revision C.01; Gaussian Inc.: Wallingford, CT, USA, 2016.

26. Yanai, T.; Tew, D.P.; Handy, N.C. A new hybrid exchange-correlation functional using the Coulomb-attenuating method (CAM-B3LYP). Chem. Phys. Lett. 2004, 393, 51-57. [CrossRef]

27. Tawada, Y.; Tsuneda, T.; Yanagisawa, S.; Yanai, T.; Hirao, K. A long-range-corrected time-dependent density functional theory. J. Chem. Phys. 2004, 120, 8425-8433. [CrossRef] [PubMed]

28. Zhao, Y.; Truhlar, D.G. Comparative DFT Study of van der Waals Complexes: Rare-Gas Dimers, Alkaline-Earth Dimers, Zinc Dimer, and Zinc-Rare-Gas Dimers. J. Phys. Chem. A 2006, 110, 5121-5129. [CrossRef]

29. Sekino, H.; Bartlett, R.J. A linear response, coupled-cluster theory for excitation energy. Int. J. Quantum Chem. 1984, 26, 255-265. [CrossRef]

30. Stanton, J.F.; Bartlett, R.J. The equation of motion coupled-cluster method. A systematic biorthogonal approach to molecular excitation energies, transition probabilities, and excited state properties. J. Chem. Phys. 1993, 98, 7029-7039. [CrossRef]

31. Hättig, C.; Weigend, F. CC2 excitation energy calculations on large molecules using the resolution of the identity approximation. J. Chem. Phys. 2000, 113, 5154-5161. [CrossRef]

32. Trofimov, A.; Krivdina, I.; Weller, J.; Schirmer, J. Algebraic-diagrammatic construction propagator approach to molecular response properties. Chem. Phys. 2006, 329, 1-10. [CrossRef]

33. TURBOMOLE V7.4 2019, a Development of University of Karlsruhe and Forschungszentrum Karlsruhe GmbH, $1989-2007$. TURBOMOLE GmbH, Since 2007. Available online: http:/ / www.turbomole.com (accessed on 11 August 2021).

34. Domcke, W.; Yarkony, D.R.; Köppel, H. Conical Intersections; World Scientific: Singapore, 2004. [CrossRef]

35. Meyer, H.D.; Manthe, U.; Cederbaum, L. The multi-configurational time-dependent Hartree approach. Chem. Phys. Lett. 1990, 165, 73-78. [CrossRef]

36. Manthe, U.; Meyer, H.; Cederbaum, L.S. Wave-packet dynamics within the multiconfiguration Hartree framework: General aspects and application to NOCl. J. Chem. Phys. 1992, 97, 3199-3213. [CrossRef]

37. Beck, M.; Jäckle, A.; Worth, G.; Meyer, H.D. The multiconfiguration time-dependent Hartree (MCTDH) method: A highly efficient algorithm for propagating wavepackets. Phys. Rep. 2000, 324, 1-105. [CrossRef]

38. Worth, G.A.; Beck, M.H.; Jäckle, A.; Meyer, H.D. The MCTDH Package, Version 8.5. 2019. Available online: http://mctdh.uni-hd de (accessed on 21 April 2021).

39. Köppel, H.; Domcke, W.; Cederbaum, L.S. Multimode Molecular Dynamics Beyond the Born-Oppenheimer Approximation. In Advances in Chemical Physics; John Wiley \& Sons, Ltd.: Amsterdam, The Netherlands, 1984; pp. 59-246. [CrossRef] 